\title{
Determinación de los niveles de iluminación de diferentes tipos de faros en un vehículo
}

\section{Determination of the levels of illumination of different types of headlights in a vehicle}

Daniela Alexandra Jerez Mayorga

Universidad Internacional del Ecuador, Ecuador

José Andrés Castillo Reyes

Universidad Internacional del Ecuador, Ecuador

Autor para correspondencia: djerez@uide.edu.ec; acastillo@uide.edu.ec

Fecha de recepción: 08 de Julio 2017 - Fecha de aceptación: 15 de Enero de 2018

Resumen: El objetivo de este proyecto es analizar los niveles de iluminación de 5 diferentes tipos de Vehículo. En su análisis comprobaremos el análisis de costos de los faros y las luces altas y bajas de los 5 vehículos. Para este análisis de medidas de luz utilizaremos una APP (aplicación) llamado "Lux" que es un programa que verifica la intensidad de la luz que se encuentra en el ambiente y utilizando un sensor del celular. Para la utilización se la APP, se apreciaría mejor en la noche es más seguro al tener un mejor sistema de iluminación.

Palabras Clave: luces; automatizadas; seguridad; iluminación

\begin{abstract}
The objective of this project is to analyze the lighting levels of 5 different types of Vehicle. In your analysis we will check the cost analysis of the headlights and the high and low lights of the 5 vehicles. For the analysis of light measurements, it uses an APPLICATION (application) called "Lux" which is a program that verifies the intensity of the light that is in the environment and uses a cell sensor. For the application is the APPLICATION, it is best appreciated at night for more security to have a better lighting system.
\end{abstract}

Key Words: lights; automated; security; illumination 


\section{Introducción}

Debido a los altos índices de accidentes de tránsito ocasionados por la falta de visibilidad en ambientes sin iluminación, se considera importante realizar la investigación sobre los niveles de iluminación de los faros automotrices actuales.

Este estudio es de suma importancia para las leyes de tránsito de cada país ya que con esto se puede saber si el vehículo pasa o no las pruebas realizadas por las agencias de tránsito de cada país. En el caso del Ecuador y específicamente en Guayaquil lo realiza la ATM (agencia de tránsito municipal) quienes ya tienen establecidos parámetros bajo los cuales se rigen.

La app la cual es objeto de estudio en este texto, se la puede encontrar en la Play Store de Android bajo el nombre de "Luxómetro" la cual ha sido seleccionada por que tiene más de cien mil descargas y tiene una calificación de 4.2/5 lo que nos pareció interesante. Esta aplicación nos permite saber la intensidad en luxes de los faros de nuestros vehículos, es decir que las personas que no poseemos un Luxómetro profesional en nuestros hogares podríamos obtener un aproximado del valor de la intensidad de nuestros faros para con esto buscar corregir si es que existen errores o reparar o también cambiar los faros si fuera el caso.

\section{Materiales y Métodos}

Para realizar el estudio de luminosidad hemos escogido 5 diferentes vehículos que son: Chevrolet Optra, Chevrolet Aveo Family, Chevrolet Forsa 2, Chevrolet Blazer, Chevrolet Spark. En la prueba de luminosidad, se utilizó un APP llamado "Lux" que es una aplicación de un sistema Android, que realiza las medidas de intensidad de luz a diferentes distancias y condiciones de luminosidad.

Se elaboró una caja de cartón para colocar dentro de ella el celular y así captar la luz de los faros del vehículo. En la medición del ancho y el alcance de luz se utilizó un flexómetro para poder colocar la caja de cartón a una distancia adecuada para recoger con exactitud los datos que genera en cada tipo de luz en bajas y altas de los 5 vehículos.

El Luxómetro para medir la iluminancia en lux (lx) o en vela (fc) con el sensor de luz. Una aplicación simple para medir la intensidad de la luz. Monitorea y compara la iluminancia de diferentes fuentes de luz. Los valores medidos dependen de la precisión del sensor de luz. Se puede diferir de la iluminancia real y entre los diferentes dispositivos. La aplicación solo puede funcionar si el smartphone tiene el sensor de luz.

* Unidad: lux o vela (foot-candle)

* Valor mínimo y máximo

* Diagrama con valor medio

* Regular la sensibilidad de sensor

* Calibrar si los valores son diferentes de los valores reals

* Cambiar de color

* Más ajustes

* Mover a la tarjeta SD 


\section{Grabación}

Graba la iluminancia de hasta 16 h 39 min. Selecciona la duración y el número de valores. Guarda y exporta los valores de la luz.

\section{Definiciones}

Lu: El lux (símbolo lx) es la unidad derivada del Sistema Internacional de Unidades para la iluminancia o nivel de iluminación. Equivale a un lumen $/ \mathrm{m}^{2}$. Se usa en la fotometría como medida de la iluminancia, tomando en cuenta las diferentes longitudes de onda según la función de luminosidad, un modelo estándar de la sensibilidad del ojo humano a la luz.

App: una aplicación móvil, o app (acortamiento del inglés application) es una aplicación informática diseñada para ser ejecutada en teléfonos inteligentes, tabletas y otros dispositivos móviles y que permite al usuario efectuar una tarea concreta de cualquier tipo — profesional, de ocio, educativas, de acceso a servicios, etc. - , facilitando las gestiones o actividades a desarrollar.

Por lo general, se encuentran disponibles a través de plataformas de distribución, operadas por las compañías propietarias de los sistemas operativos móviles como Android, iOS, BlackBerry OS, Windows Phone, entre otros (J.J. Kavanagh, R.S. Barrett, S. Morrison).

Luxómetro: Un luxómetro (también llamado luxómetro o light meter) es un instrumento de medición que permite medir simple y rápidamente la iluminancia real y no subjetiva de un ambiente. La unidad de medida es el lux (lx). Contiene una célula fotoeléctrica que capta la luz y la convierte en impulsos eléctricos, los cuales son interpretados y representada en un display o aguja con la correspondiente escala de luxes.

\section{Resultados}

Los resultados obtenidos se dividen dependiendo del tipo de vehículo que se seleccionó para realizar las pruebas, los cuales se detallan en la tabla $\mathrm{N}^{\circ} 01$.

\section{Prueba de intensidad de Luz}

Para las pruebas de eficacia del sistema se utilizó una app llamado "Lux" con el que se mide la intensidad luminosa en diferentes condiciones (L.M. Moreno). Las medidas que arrojo la app "lux" en sistema de luces altas:

- Chevrolet Optra: 4,933 1x

- Chevrolet Forza 2: 2,030 1x

- Chevrolet Aveo: 2,100 1x

- Chevrolet Blazer: 3,019 1x

- Chevrolet Spark: 2,978 


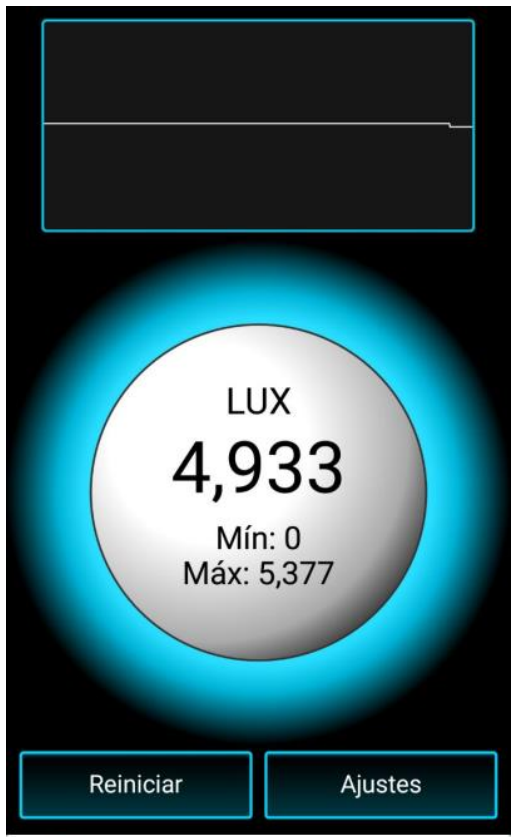

(i) $\cong$ Spotify Music

Figura 1: Medida del Chevrolet Optra en luces altas Fuente: Los autores

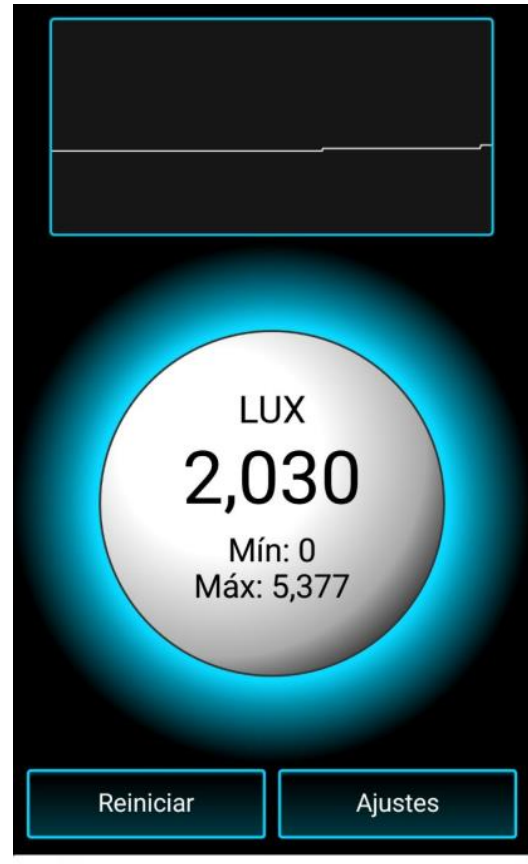

(i) $\approx$ Spotify Aplicación Aplicación Gratuita

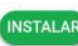

Figura 2: Medida del Chevrolet Forza 2 en luces altas Fuente: Los autores 
Tabla Nº1. Medición de luxes en los faros

\begin{tabular}{|c|c|c|c|c|}
\hline & Derecha & & Izquie & \\
\hline Vehículo & alta & baja & alta & baja \\
\hline Chevrolet Optra & $4,9331 \mathrm{~lx}$ & 2,302 & 3,820 & $1,3901 \mathrm{x}$ \\
\hline $1.5 \mathrm{~L}(2006)$ & & lx & $1 \mathrm{x}$ & \\
\hline $\begin{array}{l}\text { Chevrolet Forza } 2 \\
\text { 1.3L (2003) }\end{array}$ & $2,0301 \mathrm{~lx}$ & $1 x^{1,474}$ & $1 x^{1,642}$ & $1,1421 \mathrm{x}$ \\
\hline $\begin{array}{l}\text { Chevrolet Aveo } \\
\text { 1.5L (2011) }\end{array}$ & $2,1001 x$ & $5521 x$ & $1 x^{3,456}$ & $1,1421 \mathrm{x}$ \\
\hline $\begin{array}{l}\text { Chevrolet Blazer } \\
\text { 3.5L (1999) }\end{array}$ & $3,0191 \mathrm{x}$ & $\mathrm{lx}^{2,341}$ & $1 x^{2,744}$ & $1,1011 \mathrm{x}$ \\
\hline $\begin{array}{l}\text { Chevrolet Spark } \\
\text { 1.0L (2006) }\end{array}$ & $2,9781 x$ & $\operatorname{lx}^{1,164}$ & $1 x^{2,560}$ & $1,3291 \mathrm{x}$ \\
\hline
\end{tabular}

Fuente: Los autores

En las pruebas tomadas en vehículos de la marca Chevrolet pudimos observar que 4/5 oscilan entre 2,000 a 2,700 luxes (1x) con la excepción de uno que rodeaba los 4,900 lx, esto en intensidad alta, en cuanto a la intensidad baja hay una gran diferencia entre todos, pues hay una oscilación de entre 500 a 2,401 luxes (lx), todos estos datos paréntesis y alineándola con el margen derecho. La ecuación debe estar centrada.

\section{Conclusiones}

Notamos que, aunque el estudio fue realizado en una sola marca de vehículos, todas las lecturas que nos demostró la aplicación de luxómetro en el smartphone fueron diferentes, muy posiblemente por el desgaste de los filamentos de los faros de los vehículos estudiados. Pudimos notar que la aplicación nos permitía ver los resultados en 2 parámetros diferentes m, lux (lx) y vela (vl). La aplicación era calificada de manera muy positiva por la comunidad de Android los cuales son los que realizan sus descargas de la Play Store.

\section{Bibliografía}

Barrientos, L. Peñin, et. at (2007). "Coordenadas" en Fundamentos de Robótica, 2da ed., vol. 2, Ed. McGraw-Hill, España, pp. 217-29.

Basantes, J., Torres, F. (2009). "Desarrollo de un Sistema de Control para un Brazo Robótico mediante Adquisición y Procesamiento de Imágenes” Proyecto de titulación, Escuela Politécnica Nacional, Quito, Ecuador.

Reber, R., Mitchell, R. L, y Carter, C. J. (1968). "Oxygen absorption in the Earth's atmosphere," Aerospace Corp., Los Angeles, CA, Tech. Rep. TR-0200 (4230-46)-3.

Kavanagh, J.J., Barrett, R.S., Morrison, S. (2004). "Upper body accelerations during walking in healthy young and elderly men” Gait Pos vol. 20, pp. 291-298.

Brusberg M. A., and Clark, E. N. (1995). "Installation, operation, and data evaluation of an oblique-incidence ionosphere sounder system," in "Radio Propagation Characteristics of the Washington-Honolulu Path," Stanford Res. Inst., Stanford, CA, Contract NOBSR87615, Final Rep. vol. 1 
Moreno, L.M. (2005). "Computación paralela y entornos heterogéneos," Tesis doctoral, Dep. Estadística, Investigación Operativa y Computación, Universidad de La Laguna, La Laguna.

Newman, S.M. (1992). "Active damping control of a flexible space structure using piezoelectric sensors and actuators” Master Thesis, U.S. Naval Postgraduate School.

Rafferty, W (1994). “Ground antennas in NASA's deep space telecommunications," Proc. IEEE vol. 82, pp. 636-640.

Riess, J., Abbas, J. J. (2001). "Adaptive control of cyclic movements as muscles fatigue using functional neuromuscular stimulation”. IEEE Trans. Neural Syst. Rehabil. Eng vol. 9, pp.326-330.

Ruiz, L. I., García, A., García, J., Taboada. G. (2008). "Criterios para la optimización de sistemas eléctricos en refinerías de la industria petrolera: influencia y análisis en el equipo eléctrico," IEEE CONCAPAN XXVIII, Guatemala

Stein, L. (2004). "Random patterns," in Computers and You, J. S. Brake, Ed. New York: Wiley, pp. 55-70.

Vázquez, R. (2009). Presentación curso “Realidad Virtual”. National Instruments. Colombia.

Young, G. O. (1964). "Synthetic structure of industrial plastics" in Plastics, 2nd ed., vol. 3, J. Peters, Ed. New York: McGraw-Hill, 1964, pp. 15-64. 